\title{
Die mittelalterliche Geschichte und ihre wichtige Position in den globalhistorischen und postkolonialen Diskursen der Gegenwart
}

Schon längere Zeit mehren sich die Stimmen in der Mediävistik, die für eine stärkere Öffnung des Fachs über seine übliche Perspektivenbeschränkung auf Europa hinaus plädieren und eine Ausrichtung hin zu einer Bestimmung der Rolle der Zeit vor 1500 im Rahmen der größeren Globalgeschichte anstreben. Dies ist zunächst vor allem durch eine neue Konzentration auf den Mittelmeerraum geschehen, der gerade den Handlungsradius der Kaufleute aus italienischen Seerepubliken, allen voran Venedig und Genua, erneut in den Fokus der Forschung gerückt hat. Zudem erkennt man auch einen neuen Blick der Mediävistik auf Eurasien, wie ihn etwa Hermann Kulke eindringlich forderte - ein Blick, der insbesondere das Wissen um Asien in Europa, die spätmittelalterliche Kartographie, die franziskanische Mission und die Asienreiseberichte neuerlich zum Thema der Forschung machte. Die Interaktion mit der islamischen und byzantinischen Welt erfuhr ebenfalls eine größere Aufmerksamkeit, doch entstand hier jüngst auch der Widerspruch gegen eine Verwendung oder Ausdehnung des Mittelalter-Begriffes an sich für Kulturkreise außerhalb Lateineuropas (Thomas Bauer). Das verbindet sich mit dem zugleich spürbaren Unbehagen in der Zunft, das Mittelalter aus seinem europäischen Kontext zu lösen. Eine in der Quellenkunde besonders bewanderte Teilepoche der Geschichte wird aus guten Gründen besonders vorsichtig und bedacht mit einem Perspektivwechsel umgehen, der den Blick von außen auf Europa wirft und damit auch neue Sprach- und Quellenkompetenzen erfordert, die nur in interdisziplinärer Perspektive zu erlangen sind. Die Kooperation mit der Sinologie und Indologie haben hier bereits erste Wege eröffnet.

Ein aktueller Beitrag von Geraldine Heng versucht nun mit einer - trotz des beachtlichen Umfangs des Buches - geradezu essayhaften Klarheit, die Mediävistik auf diesem langsamen Weg in eine Neubewertung des europäischen Mittelalters in der Globalgeschichte postkolonialen Zuschnitts zu verorten. Dabei möchte sie eine neue Ebene der Diskussion erreichen, indem sie eines der überraschend lange weitgehend unhinterfragt wiederholten Urteile über das Mittelalter aufgreift - nämlich, dass es in dieser Epoche keinen Rassismus im modernen Sinne gegeben habe. Geraldine Heng nimmt nun pointiert die Gegenposition ein und verlängert die Vorgeschichte des

Rezension von: Geraldine Heng, The Invention of Race in the European Middle Ages, Cambridge etc. (Cambridge University Press) 2018, XIII, 493 S., Abb., ISBN 978-1-108-42278-9, GBP 34,99.

Kontakt: Romedio Schmitz-Esser, romedio.schmitz-esser@zegk.uni-heidelberg.de 
Rassismus bis ins Hochmittelalter. Nach einer theoretisch fundierten Zurückweisung dieses Vorurteils, das das europäische Mittelalter gleichsam gegen einen wichtigen und sehr berechtigten moralischen Vorwurf verteidigt hätte, fährt das Buch mit der Vertreibung der Juden im hochmittelalterlichen England fort, um dann die Konzepte von Sarazenen, den Diskurs über Menschen mit schwarzer Hautfarbe, die Einstellungen zu den Ureinwohnern Nordamerikas (insbesondere in der Besiedlung Islands, Grönlands und Neufundlands durch die Wikinger), die Behandlung der Mongolen und schließlich der Roma in den Blick zu nehmen. Die damit angesprochenen sieben Kapitel des Buches ergeben zusammen ein Bild, das die wichtige Diskussion über den Begriff des Rassismus und seiner tiefen historischen Wurzeln in der europäischen Kultur aufgreift und zum Thema macht. Dieser postkoloniale Blick ist nicht nur begrüßenswert, er ist auch wichtig für die Mediävistik allgemein, denn er kennzeichnet die Epoche als eine zentrale Achse in unserem Verständnis der globalhistorischen Entwicklung.

Die Analyse der Einzelkapitel erweist dabei trotz des großen Umfangs nur wenig Neues und orientiert sich größtenteils am Forschungsstand, was sich auch dadurch erklären mag, dass sich das Buch ausdrücklich auch an eine breitere Leserschaft wendet. Die Innovation von Hengs Ansatz liegt aber eben darin, diesen Forschungsstand in den genannten Themengebieten für das Hoch- und Spätmittelalter gezielt hin auf rassistische Konzepte zu lesen. Dreh- und Angelpunkt wird damit die Frage nach der Definition von „race“ selbst; wenn man diesen notorisch unscharfen - weil eben eigentlich etwas Nicht-Existentes, sondern kulturell und sozial Konstruiertes bezeichnenden - Begriff über ein rein biologistisches Verständnis ausweitet, in dem kulturelle Konstruktion des Anderen über Geschlecht, Ethnizität und religiöse Differenz eine wichtige, freilich fluktuierende Rolle spielt, dann wird man die Vorgeschichte des Rassismus weit in das Mittelalter zurückführen können. Damit weist Heng der Mediävistik einen Anteil am globalhistorischen Diskurs zu, aus dem diese Epoche völlig zu Unrecht und oft selbstverschuldet - zu leicht ausgeklammert wird. Gibt man einmal den geistigen Widerstand auf, „race“ nur in einem biologistischen Rahmen $\mathrm{zu}$ verstehen (wofür es ausgezeichnete Gründe gibt), dann könnte man eher umgekehrt dem „Götzen Ursprung“ noch weiter folgen und würde sicherlich auch etwa in der Antike und im Frühmittelalter weitere, ältere europäische Rassismen finden, die zudem in der spätmittelalterlichen Beschreibung Asiens noch erheblich weiterwirkten, etwa beim Kannibalismus-Vorwurf gegenüber den Mongolen. Auch Beschreibungen von Hunnen oder Awaren, Ungarn oder Wikingern ließen sich beispielsweise entsprechend für das Frühmittelalter oder die Spätantike ergänzend untersuchen. In diesem Licht erkennt man aber auch, dass die zeitliche Veränderung, die Heng etwa im Kapitel über „Epidermal Race“ anspricht, noch größere Aufmerksamkeit für die künftige Diskussion verdient. Letztlich dominiert das Buch jedoch ein der Globalgeschichte latent innewohnender teleologischer Impetus, der die moderne Welt als Gipfelpunkt der Globalisierung sieht und deren auf diesen Zustand zulaufende Vorgeschichte zu verstehen trachtet. Das Mittelalter könnte aber vielleicht gerade eine 
Epoche sein, in der die wellenartigen Vor- und Rückwärtsbewegungen in diesem Prozess noch einmal deutlich greifbar werden.

Der Aufforderung der Autorin nach einer Diskussion folgend, sollte eine Reflexion dieses fast 500 Seiten umfassenden Werkes also auch in dessen Kritik einsteigen. Die wenig innovative und oft eher stereotype Behandlung der konkreten Schriftquellen und Bildzeugnisse lenkt mitunter eher von der zentralen Stoßrichtung des Buches ab, weshalb eine Kritik hier aber auch bis zu einem gewissen Grad am wesentlichen Kern des Buches vorbeizielen würde. Hengs Buch blendet gegenläufige Befunde allerdings nicht aus, sondern lässt sie vielmehr stehen, etwa wenn sie auf das Beispiel der dunkelhäutig dargestellten Königin von Saba am Verduner Altar und vor allem auf die Statue des Heiligen Mauritius aus Magdeburg eingeht. Mauritius, der offensichtlich ein positives Bild eines Afrikaners in Europa darstellt, ist für sie ein Mysterium (,the extraordinary enigma of the Black St. Maurice of Magdeburg“, S. 242); und tatsächlich greift ihre Überlegung, dass es vor allem um eine Rechtfertigung des imperialen Anspruches auf allumfassende Herrschaft (auch) in Afrika im Nachgang des Fünften Kreuzzugs ginge, wohl zu kurz, denn damit sind die komplexen Vorgänge in Magdeburg und die besondere Beziehung des Hofes Friedrichs II. zur islamischen Welt doch nur unzureichend angesprochen. Allerdings vermeidet Heng hier wie andernorts auch ein zusammenfassendes Urteil, das sie lieber ihren Leserinnen und Lesern überlässt. Tatsächlich fehlt dem Buch ein Schlusswort oder eine Zusammenfassung, was die erwünschte Öffnung für eine breitere Diskussion sicherlich symbolisiert; man darf aber doch fragen, ob diese dadurch erleichtert wird. $\mathrm{Zu}$ den Herausforderungen der aktuellen Mediävistik gehört schließlich auch ein immer breiterer, interdisziplinärer Diskurs, der übrigens längst die hier gestellten Fragen ebenfalls berührt hat. So kommt der archäologische Befund in dieser Reflexion nicht vor, obwohl es mittlerweile mit dem mittels neuer naturwissenschaftlicher Techniken untersuchten „Ipswich Man“ durchaus Hinweise auf ein gleichberechtigtes Zusammenleben von Menschen afrikanischer Herkunft sogar in England gibt. Man darf solche Funde sicher nicht überbewerten, doch wenn es um ein komplexeres, vollständigeres Bild des Mittelalters in der hier behandelten Frage geht, so brauchen wir eben auch möglichst viele der verfügbaren Quellen in unserer Diskussion. Dass die unterschiedlichen Fachdiskurse der mit dem Mittelalter beschäftigten Disziplinen in Zukunft über Lippenbekenntnisse hinweg noch enger zusammenarbeiten müssen, zeigt Hengs Buch in besonders deutlicher Weise auf; sie selbst verbindet hier verdienstvoll sprach- und literaturwissenschaftliche mit historischen Ansätzen.

Deshalb scheint mir auch gewichtiger als jeder kleinteilige, inhaltliche Einwand, der die Einordnung einzelner Quellen betrifft, der Verweis auf die Schwierigkeiten einer Ausrichtung des postkolonialen Arguments auf einen spezifischen, zeitgenössischen und weitgehend englischsprachigen Diskurs. Ein zentrales Problem stellt sich so schon bei der Übertragung des Begriffes „race“ in den Diskurs außerhalb der anglophonen Welt; es versteht sich, dass man etwa im Deutschen wohl kaum unproblematisch von einer jüdischen „Rasse“ sprechen würde - doch gerade das ist 
ja eine wichtige Voraussetzung, um die (wiederum nur für das Königreich England) untersuchte Ausgrenzung von Juden im Mittelalter als Vorgeschichte des modernen Rassismus erzählen zu können, wie es Heng vorschlägt. Die durchaus erwünschte soziokulturelle Rückbindung des Werkes von Heng an ihre eigene Gegenwart limitiert somit die Übertragbarkeit der Ergebnisse auf das gesamte Mittelalter. Das heißt allerdings gerade nicht, dass die Diskussion, die hier angestoßen wird, nicht auch die kontinentaleuropäische Mediävistik beträfe; im Gegenteil, die Diskussion hat hier erst begonnen. Die Arbeit lässt sich nämlich in eine Linie mit einem breiteren Forschungstrend stellen, in dem in den letzten Jahren Themen wie die mittelalterliche Sklaverei und die Geschichte Afrikas im Mittelalter in den Fokus der Forschung getreten sind. Umso bedauerlicher ist es, dass ein (auch auf Englisch übersetztes) einschlägiges Werk wie François-Xavier Fauvelles „Rhinocéros d’or“ in der Bibliographie ebenso fehlt wie Marina Münkelers „Erfahrung des Fremden“ und Folker Reicherts oder Felicitas Schmieders Arbeiten zu den Ostasienreiseberichten. Hier zeigt sich auch der bibliographische Fokus auf die englischsprachige Forschung als ein Hindernis für einen breiteren Blick auf das Thema - eine eigene Form des Überlebens kolonialen Denkens in einseitiger Sprachdominanz, die in diesem Beitrag zu den postkolonialen Studien etwas merkwürdig anmutet.

Es ist insbesondere die Geschichte der italienischen Halbinsel mit ihrer zentralen Funktion als Drehscheibe des Handels und des Wissensaustauschs für den nordalpinen Raum, durch den Europa im Mittelalter mit Afrika und Asien in Kontakt kam. Die von Geraldine Heng angestoßene Diskussion ist Teil einer weit breiteren Neuausrichtung der Mediävistik insbesondere in der letzten Dekade, deren weitere Entwicklung dieser Epoche ihren wichtigen Platz in der globalhistorischen Diskussion zuweisen wird. Stärke und Schwierigkeiten dieses Weges werden hier zugleich deutlich: Die Stärkung der Relevanz mediävistischer Studien, auch und insbesondere für einen breiteren, zeitgenössischen Diskurs; die Notwendigkeit, sehr heterogene Fachdiskurse mit verschiedenen sprachlichen Kontexten in ein produktives Gespräch zu bringen; und schließlich der eingehende Blick auf eine Welt, die sich nicht einfach als geradlinige Vorgeschichte unserer Gegenwart verstehen lässt, mit dieser aber in engster Beziehung steht. Dieses Spannungsfeld sichtbar zu machen und eine Diskussion über diese Punkte zu befördern, ist die besondere Stärke von Hengs Buch. 